\title{
Blockchain Technology and its Impact on the Global Economy
}

\author{
Dr. Burcu Sakız (Istanbul Aydın University, Turkey) \\ Prof. Dr. Ayşen Hiç Gencer (Beykent University, Turkey)
}

\begin{abstract}
The world's most valuable resource is no longer oil, but data. Smartphones and the internet have made data abundant, ubiquitous and far more valuable. Modern algorithms can predict when a customer tends to buy, a car needs servicing or a person is at risk of a disease. Meanwhile, artificial intelligence techniques extract more value from data. As individuals accumulate information which transforms into knowledge, entrepreneurs will want to use and/or share that knowledge. It is the sharing of knowledge that needs a decentralized, autonomous mechanism so that knowledge can be shared fairly amongst all peoples of the world, not just within corporations. Blockchain technology gives us that mechanism. Blockchain is one of a kind decentralized technology and it is distributed as well as decentralized ledger. Blockchain is the answer to a lot of obstacles the world has to go through today. Before today, nobody could think of transferring money from one account to another safely without any financial institution in the middle, like a bank. Blockchain technology presents a radical and disruptive new way of conducting all manner of transactions over the Internet. The advent of Bitcoin and the blockchain has brought a lot of change to the world of finance even the world economy was formerly run using fiat currencies. Introducing the blockchain environment will actually enhance the economics because in blockchain, all transactions are recorded right from the manufacturer to the buyer. This paper explores the emerging landscape for blockchain technology focusing on the economics.
\end{abstract}

\section{Introduction}

Blockchain is one of the hottest topics almost all around the world. Especially with the beginning of the 21 st century, there are many major developments and changes seen in financial technologies like blockchain. What it really means and what it stands for in the global economy today is very important. Blockchain technology and distributed database technologies becomes the key technological enablers of recent developments in distributed transaction and ledger systems. These technologies cause opening of new gates for opportunities based on open sources especially new types of digital platforms and services (Lindman vd., 2017). We can define open source as they refer to any program whose source code is made available and open source software is usually developed as a public collaboration and made freely available (Url-1,2019). Usually new technologies attract a wide variety of developers especially in IT (information technologies) industry, including many freelancers from around the world. At the elementary stage of blockchain solutions, it is likely to be in a developer's best interest to develop, or monitor the development of, blockchain applications on open source. And it is going to change the way applications and users interact with the internet.

Blockchain is touted as one of the most significant technical innovations in digitalization of asset ownership in this century. The most impactful blockchain applications will require tight collaboration between developers, incumbents, innovators, and regulators, adding complexity and delaying implementation (Url-2, 2016). Blockchain environment refers to a fully distributed system for cryptographically capturing and storing a, immutable, consistent and linear event log of transactions between networked actors. It is similar to a distributed ledger that is consensually kept, updated, not deleted and validated by the parties involved in all the transactions within a network (Risius, Shoprere, 2017). Blockchain technology currently receives a lot of academic and public attention. It has an aim to constitution of the foundation for truly trust-free economic transactions (Glaser 2017). Normally blockchain systems can not only process monetary transactions but can also ensure that transactions comply with programmable rules in the form of "smart contracts" (Tschorsch, Scheuermann 2016), it allows even parties to conduct and reliably control mutual transactions without requiring of any trusted middle-men.

Blockchain can be described as "the trust machine," indicating that it takes care of trust issues between individuals (Url-3, 2019). It is an open source ledger that is visible to users across the network brings a level of security that is unmatched. Blockchain puts the trust in its users and their ability to maintain the ledger. In other words, blockchain technology built economic system runs without people, thus making a transaction "trust-free". This technology provides a viable alternative to eliminate middle-mans, thereby lowering operational costs and increasing the efficiency of a sharing service. Blockchain is a platform where people are allowed to carry out transactions of all sorts without the need for a central or trusted arbitrator. With blockchain technology, the world's most fundamental commercial interactions can be re-imagined. That causes many opportunities to invent new styles of digital interactions in trust-free sharing services (Sun et al., 2016).

The blockchain, the ledger that underlies the famous cryptocurrency Bitcoin, has huge implications for many industries. The advent of Bitcoin and the blockchain has brought a lot of change to the world of finance. Also it is kind a financial tool that can potentially play an important role in the sustainable development of the global 
economy. That's why this paper focuses on the emerging landscape for blockchain technology in terms of economics.

\section{History of Blockchain Technology}

The blockchain technology promises to revolutionize the way of business. It has effects on various sectors, from financial to manufacturing as well as education. Satoshi Nakamoto released the well-known whitepaper about the technology in 2009. In the paper, he provided details of how the technology was well equipped to enhance digital trust given the decentralization aspect that meant nobody would ever be in control of anything. Ever since Satoshi Nakamoto exited the scene and handed over Bitcoin development to other core application developers, the digital ledger technology has evolved resulting in new applications that make up the blockchain history (Url-4, 2018). The evolution of Bitcoin and other cryptocurrencies have both drawn significant attention and also threatened the very foundations of the financial system. After all, this was the intention of Satoshi Nakamoto when the global financial crisis hit not only USA but also the global economy harder than any crisis in history.

But after the global financial crisis, the year 2009 is not the exact date that blockchain concept revealed. Blockchain was invented in 1991. Blockchain history dates back to early 1990's by two researchers: Stuart Haber and W. Scott Stornetta. They both touted as the co-inventor of the blockchain technology. Several aspects of the Bitcoin blockchain architecture are based on Stornetta's work. They described the concept of a cryptographically secured network of blocks.

The first mention of blockchain architecture was held in a publication that Stornetta coauthored described a digital hierarchy system known as a "block chain" that utilized digital time-stamps for ordering transactions. They worked on a cryptographically secured chain of blocks whereby no one could tamper with timestamps of documents. Afterwards they upgraded block chain system to incorporate Merkle trees that enhanced efficiency thereby enabling the collection of more documents on a single block in 1992 (Url-5,2019).

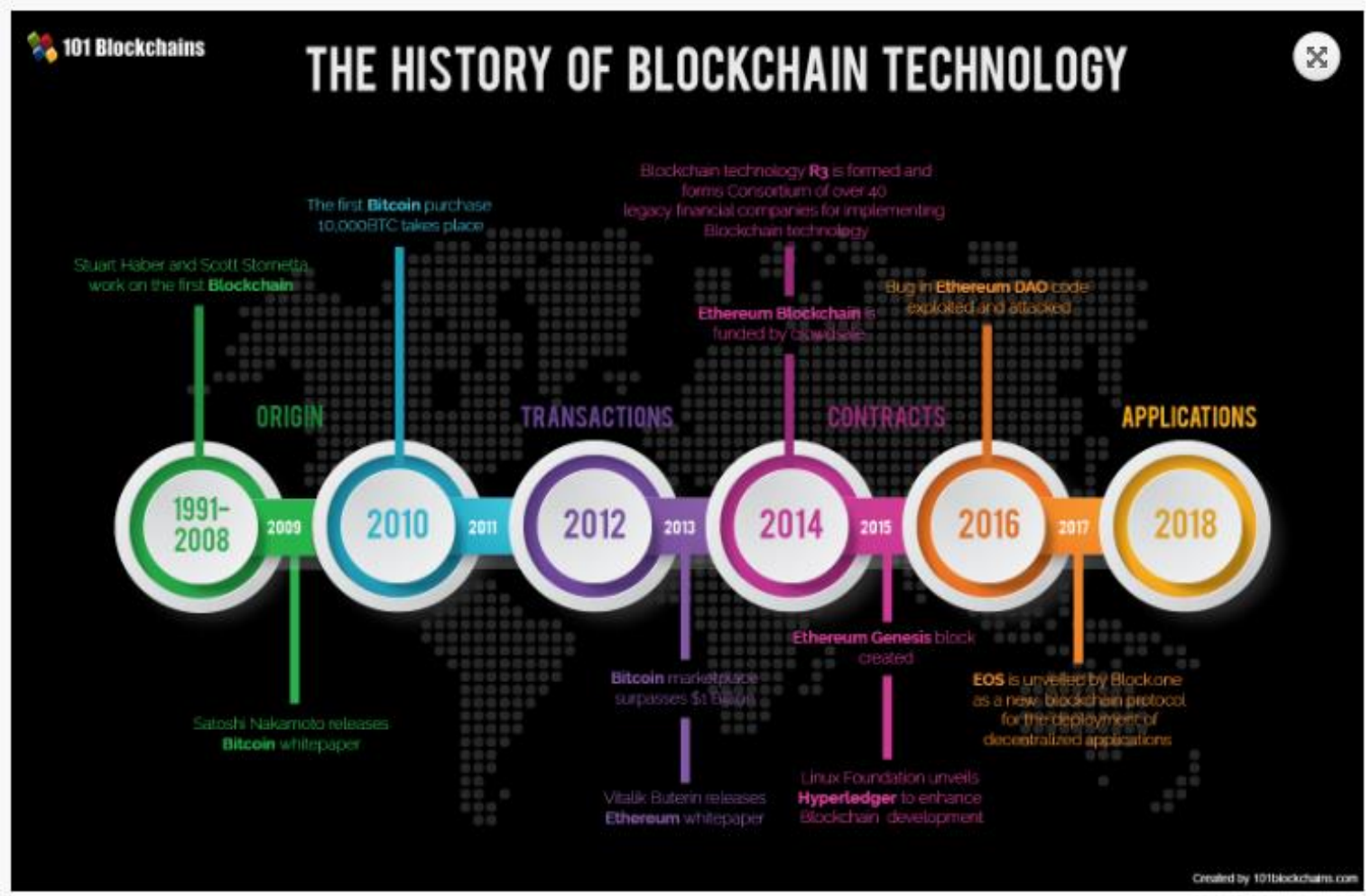

Figure 1. The History Of Blockchain Technology Source: Url-4, 2018.

Additionally, by 1998 Nick Szabo had begun working a decentralized digital currency which was called as Bit Gold. It wasn't until 2009 that developer Satoshi Nakamoto implemented the first blockchain. Nakamoto is known for creating the world's first digital currency called Bitcoin. Nakamoto is a group of developers who collaborated in order to use blockchain technology to create Bitcoin. Bitcoin utilized blockchain technology on a peer-to-peer network as a ledger for any transactions made with Bitcoin, (Url-6, 2019). Very little is known about Nakamoto as people believe whether he or she could be a person or a group of people that worked on Bitcoin, which was accepted as the first application of the digital ledger technology. Figure 1 above Blockchain history is illustrated.

Moreover, blockchain technology has several essential features developed in time. One is that it allows a deep transition from a centralized transactional model, which until today has prevailed, to a decentralized one. 
distributed system should turn out to be a more robust and reliable solution than is usually provided by a centralized authority to its stakeholders - such as a government to its citizens (Collomb, Sok, 2016 ). Table below summarized standard transactions versus blockchain ones.

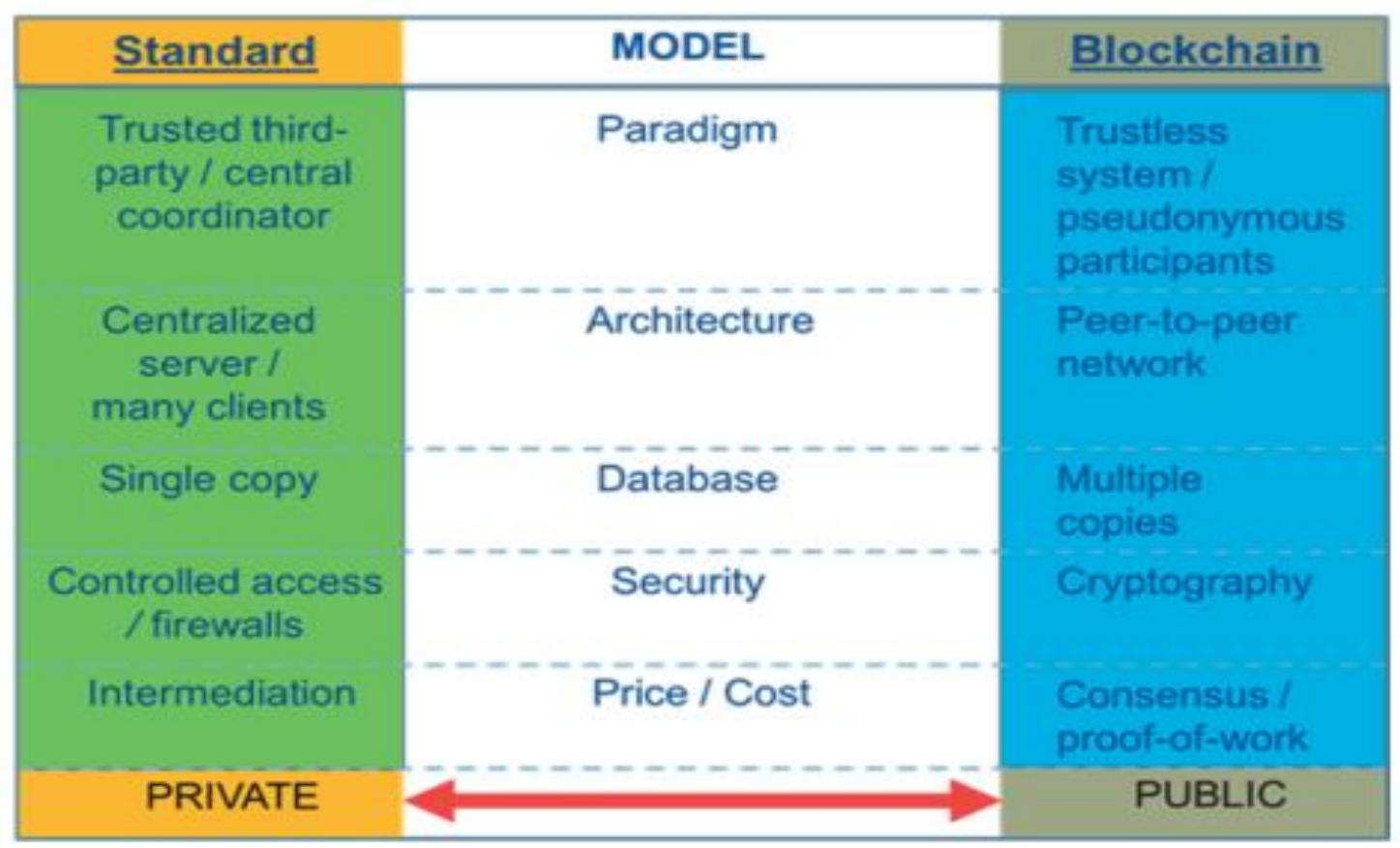

Table 1. Standard Versus Blockchain based Transactional Models Source: Collomb, Sok, 2016.

\section{Blockchain Evolution}

A blockchain's three main benefits are that it provides transparency, authentication and auditing abilities and generally evolution of blockchain technology can be held in two phases. First phase consists of years between 1991 and 2013. Actually the cost of the bankruptcy of Lehman Brothers in 2008 to the USA is estimated in trillions and unfortunately it triggered a chain of events that sent several countries into economic recession or depression. Bitcoin came into being in 2008 as the first application of Blockchain technology. One contributor to the crisis was the centralized payment and monetary system based on clearinghouses that act as intermediaries between buyers and sellers and take on the risk of defaults. Bitcoin is an innovative technology that may allow banks to settle accounts among themselves without relying on centralized entities. Based on Blockchain technology, Bitcoin is considered the first decentralized currency system that works on a global scale. It relies on cryptographic proofs of work, digital signatures, and peer-to-peer networking in order to provide a distributed ledger containing transactions (Ateniese et al, 2017). Ever since Bitcoin number of applications have cropped all of which seek to leverage the principles and capabilities of the digital ledger technology. Consequently, blockchain history contains a long list of applications that have come into being with the evolution of the technology (Url-4, 2018).

Blockchain phase 2 is called Contracts and consist of years between 2013 and 2015. Developed by Vitalik Buterin, Ethereum was born out as a new public blockchain in 2013 with extended functionalities compared to Bitcoin, a development that has turned out to be a pivotal moment in Blockchain evolution timeline. He differentiated Ethereum from Bitcoin Blockchain by enabling a function that allows people to record other assets such as contracts. With his development new features expanded Ethereum functionalities from being a cryptocurrency to be a platform for developing decentralized applications as well. In 2015, Ethereum blockchain has launched and become one of the biggest applications of blockchain technology given its ability to support smart contracts used to perform various functions (Url-4, 2018).

Also in 2015, In 2015, the Linux Foundation unveiled an Umbrella project of open source blockchain called Hyperledger. Hyperledger does not support Bitcoin or any other cryptocurrency. Hyperledger focus is to encourage the use of blockchain technology to improve performance and reliability of current systems to support global business transactions. It is something like a hub for open industrial blockchain development. (Url-7, 2019).

In 2017, a company called Eos published a paper detailing a new blockchain protocol powered by an EOS as the native cryptocurrency. s main purpose is to encourage the deployment of decentralized applications through an autonomous decentralized corporation. Phase 3 of Blockchain began in 2018 and called applications. In recent years, a number of projects have cropped up all leveraging blockchain technology capabilities. Neo is one of the applications launched in China, billed as the first open source, decentralized and blockchain platform. Neo casts 
itself as the Chinese Ethereum having already received the backing of Alibaba CEO Jack Ma. Another cryptocurrency platform is optimized for the Internet of things (Iot) ecosystem as it strives to provide zero transaction fees as well as unique verification processes. Called IOTA. Than Monero Zcash and Dash blockchains came into being as a way of addressing some issues such as security and scalability (Url-4, 2018). Blockchain evolution and milestones summarized in table below.

\begin{tabular}{|c|c|c|c|c|}
\hline Timeline & Blockchain & Bitcoin & Ethereum & NEO \\
\hline 1991-2008 & $\begin{array}{l}\text { Stuart Haber and Scott } \\
\text { Stornetta Work on The } \\
\text { First Blockchain }\end{array}$ & & & \\
\hline 2009 & & $\begin{array}{l}\text { Satoshi Nakamoto } \\
\text { Releases Bitcoin White } \\
\text { Paper }\end{array}$ & & \\
\hline 2010 & & $\begin{array}{l}\text { The First Bitcoin } \\
\text { Purchase 10,000BTC } \\
\text { take place }\end{array}$ & & \\
\hline 2013 & & $\begin{array}{l}\text { Bitcoin Marketplace } \\
\text { Surpasses } \$ 1 \text { Billion }\end{array}$ & $\begin{array}{lr}\text { Vitalik } & \text { Buterin } \\
\text { Releases } & \text { Ethereum } \\
\text { White Paper } & \\
\end{array}$ & \\
\hline 2014 & & & $\begin{array}{l}\text { Ethereum Blockchain } \\
\text { Is Funded By } \\
\text { Crowdsale }\end{array}$ & \\
\hline 2014 & $\begin{array}{l}\text { Blockchain } \\
\text { Technology R3 is } \\
\text { Formed and forms } \\
\text { Consortium of Over } 40 \\
\text { Legacy financial for } \\
\text { implementing } \\
\text { Blockchain } \\
\text { Technology }\end{array}$ & & & \\
\hline 2014 & & & & $\begin{array}{l}\text { NEO Project Is } \\
\text { Launched as Antshares } \\
\text { by Da Hongfei and } \\
\text { Erik Zhang }\end{array}$ \\
\hline 2015 & & & $\begin{array}{lr}\text { Ethereum } & \text { Second } \\
\text { Blockchain } & \text { Is } \\
\text { Unveiled } & \\
\end{array}$ & \\
\hline 2015 & $\begin{array}{lr}\text { Linux } & \text { Foundation } \\
\text { Unveils } & \text { Hyperledger } \\
\text { To } & \text { Enhance } \\
\text { Blockchain } & \\
\text { development } & \\
\end{array}$ & & & \\
\hline 2017 & $\begin{array}{l}\text { EOS.IO is Unveiled by } \\
\text { block.one as a new } \\
\text { blockchain protocol for } \\
\text { the deployment of } \\
\text { decentralized } \\
\text { applications }\end{array}$ & & & \\
\hline 2015-2018 & \multicolumn{4}{|c|}{$\begin{array}{l}\text { Blockchain Technology Continues To Evolve Depicted by increased number of cryptocurrencies } \\
\text { as well as Companies leveraging the Technology To enhance Efficiency }\end{array}$} \\
\hline
\end{tabular}

Table 2. Blockchain Evaluation Source: Url-4,2019.

\section{Impacts on Global Economy and Economic Benefits of Blockchain}

Blockchain can be defined as a chain of blocks of information, called digital ledgers. These ledgers are chronologically linked and replicated not in a centralized database but in a distributed database. Information can be added as blocks and never deleted and also any change is monitored and validated by the chain Each block is protected by cryptographic algorithms, and only authorized ones can access the information. Although private blockchains exist, a typical blockchain is public and identified as "decentralized". The four main kinds of blockchain applications are money transfer and payments, property registries, contractual agreements, and identity confirmation. Replacing the dependency on trust with cryptography means that most verification, identification, 
authentication, and similar forms of assurance, accreditation, certification, and legalization of identity, origin, competence, or authority of persons or assets can be assured by mathematics (Ljutic, McPhee, 2017).

In another words, Blockchain (distributed ledger technology) is a network software protocol that enables the secure transfer of money, assets, and information via the Internet, without the need for a third-party intermediary such as a bank. A blockchain can be used as a digital registry to record, transfer, and verify asset ownership (such as home, auto, stocks, bonds, mortgages, and insurance etc.), and also can be used to preserve the integrity and authenticity of sensitive documents or records (e.g., passports, visas, driver's licenses, birth and death certificates, voter registration, contracts, wills, patents, and medical records). In a network economy with blockchain based asset transfer, personalized financial and government services might be better tailored to individual needs. With Blockchain technology, many daily operations involving money, assets, and documents could start to be conducted on digital networks with the help of cryptographic security algorithms. Given that less friction and human involvement may be needed to transfer goods and services, less physical infrastructure might be needed to make it happen (Swan, 2017).

It is widely accepted that the computational architecture of blockchain technology (distributed ledger technology) creates a wide range of potential uses. For instance, by providing an immutable, distributed ledger, it can help to facilitate not only peer-to-peer payments, but also manage records, track physical objects and transfer value via smart contracts, all without a third party or manual reconciliation. Developments in computer processing power and networked computer systems have facilitated advances in blockchain applications, while the domination of smartphones has made digital wallets possible and increasingly relevant. Additionally, there has been a proliferation of IoT (internet of things) and AI (artificial intelligence) applications that can automate bigdata collection and processing for use in blockchain platforms (Wef, 2018).

Blockchain applications are commonly used with cryptocurrencies i.e. currencies that use public-key cryptography as security measure and to prevent counterfeiting transactions. Blockchain technology can be seen both as a technical and as an economic innovation (Liebenau, Elaluf-Calderwood, 2016). Blockchain technology (distributed ledger) can be utilized as a transactional mechanism for "sharing economy" services, as it solves trusted recording of large-scale peer-to-peer activities naturally. Blockchain as an economic innovation offers solutions where there exists a need for a reliable record of transactions in a decentralized environment where not all parties can be fully trusted (Mainelli, Smith, 2015). And blockchain technology has created a global economy of immediate trust and value, built on an agreement and complex computer algorithms. Also digitized streaming money and payment channels could be techniques to quicken the 30-60-90 day terms and uncollectible debt problem in supply chain finance, and facilitate a just-in-time economy for money (Swan, 2017).

When it comes to Blockchain economy, it can be defined as a term for a move toward cryptocurrencies and digital ledger systems, and away from traditional national hard currencies and legacy, old fashioned ledger systems. In the blockchain economy, technologies like bitcoin and blockchain are the typical tools for financial management, rather than traditional software application programs managing existing national currencies. Also the blockchain economy is a scenario and potential future environment that cryptocurrencies will replace current monetary systems globally (Url-8, 2018). Also with blockchain-based asset transfer, personalized financial and government services might be better tailored to individual needs. Blockchain technology currently revolutionizes the storing, management and transfer of value between digital identities in many economic sectors.

The Future of the Blockchain Market Report revealed distributed ledger technology (DLT) named blockchain optimistically would have a positive economic impact. It will contribute as much as $\$ 120$ billion dollars worldwide between 2018 and 2024. Also the study revealed that the benefits offered by blockchain meant mainstream adoption was highly probable. They predicted that blockchain could contribute anywhere between $\$ 87$ billion to $\$ 120$ billion by 2024 , depending on which industries add it and the rate of adoption (Url-9, 2018).

Blockchain technology has been holding many promises for not only the financial sector, in particular its financial markets infrastructure, but also for the insurance industry. At the heart of this enthusiasm for blockchain lies the new decentralized transactional model that the technology permits, whose principles are based upon Satoshi's white paper about peer to peer communication (2008). Blockchain technology is very likely to have a very strong impact on the digital economy and global e-commerce, precisely because of this decentralized transactional model that it facilitates. And indeed, even if the share of e-commerce has been steadily rising since the age of the Internet, essentially over the last score of years, it remains that the main transactional paradigm is centralized (Collomb, Sok, 2016).

While blockchain technology is effecting financial sector heavily, with blockchain technology, new industry leaders are emerging. Financial services seem near term future leader of blockchain. Other sectors such as energy, industrial products, healthcare and utilities. Figure below, World Economic Report experts shows proportions of sectors effected by distributed ledger technology (blockchain) with the help of PwC Global Blockchain Survey in 2018 (Wef, 2018). 


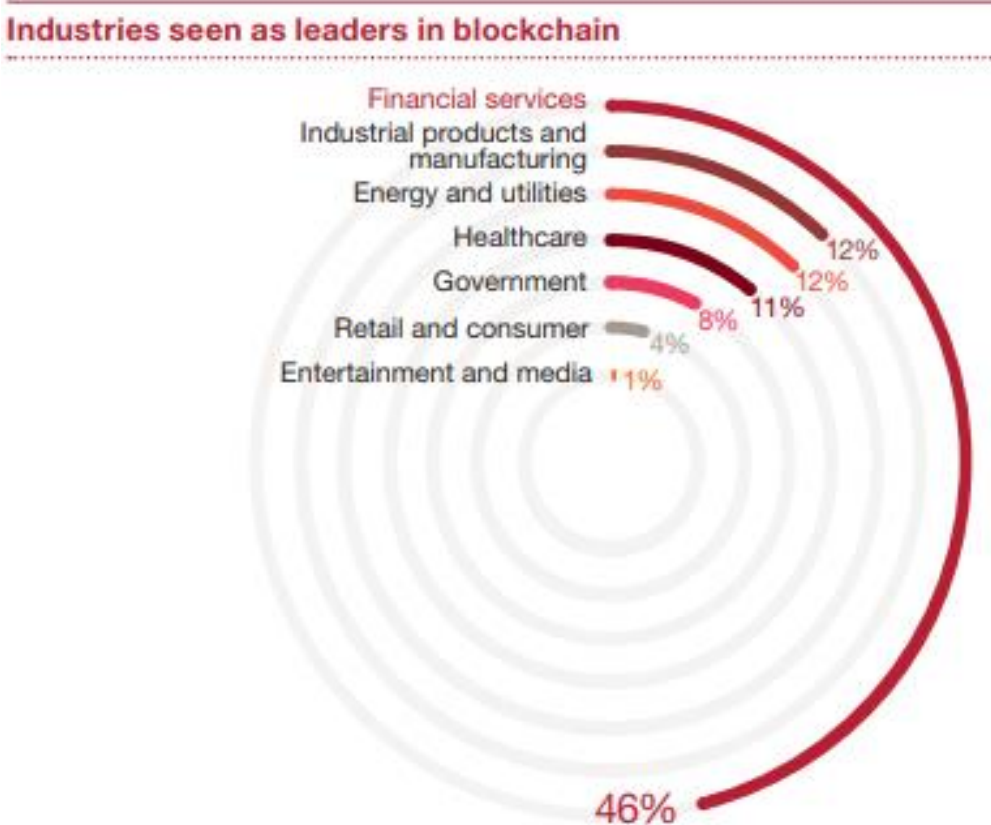

Note: Base: 600.

0 : Which of the following industries are the most advanced in developing blockchain today?

Source: PwC Global Blockchain survey, 2018

Figure 2. Industries Seen As Leaders in Blockchain Source: Wef, 2018.

According to some experts, there are four waves of anticipated blockchain deployments summarized in Table 2 below. They initially expect the first two waves to be focused on sharing and using data, before expanding to critical infrastructure once confidence in blockchain technology grows. The third wave involves the adoption of blockchain technology in major pieces of capital markets infrastructure. Truly decentralized financial ecosystem will arise in final wave, is perhaps the most ambitious and the most uncertain one (Url-10, 2019).

\begin{tabular}{|c|c|c|}
\hline Wave & Advancements & Examples in developmen \\
\hline $\begin{array}{l}1 \\
\text { Information sharing } \\
2016-19\end{array}$ & $\begin{array}{l}\text { - Blockchain used to share and communicate data } \\
\text { - Used internally and between trusted external organizations } \\
\text { - Distributed ledger solutions tested in parallel with current } \\
\text { workflows as proof of concept } \\
\text { - Augmentation of existing processes }\end{array}$ & $\begin{array}{l}\text { CDS trade } \\
\text { processing } \\
\text { Payment } \\
\text { messaging }\end{array}$ \\
\hline $\begin{array}{l}2 \\
\text { Data solutions } \\
2017-25\end{array}$ & $\begin{array}{l}\text { - Blockchain enables an environment to store and manipulate data } \\
\text { - Incorporation of distributed ledger technology as part of existing } \\
\text { solutions, supporting new efficiencies in operations and workflows } \\
\text { - Initial pilots may run in parallel with existing processes, } \\
\text { until user confidence is high enough to begin migrating volumes } \\
\text { - Users are faced with a choice of infrastructures developed by providers }\end{array}$ & $\begin{array}{c}\text { Transaction } \\
\text { management } \\
\text { Regulatory } \\
\text { reporting }\end{array}$ \\
\hline $\begin{array}{l}3 \\
\text { Critical infrastructure } \\
2020-30\end{array}$ & $\begin{array}{l}\text { - Blockchain adopted by market participants as main infrastructure } \\
\text { for critical functions } \\
\text { - Centralized authority still required for administrative functions } \\
\text { (e.g., granting access rights, setting industry standards) } \\
\text { - Replacement of existing asset, transaction and payments infrastructure } \\
\text { - Participants forced to adopt and integrate new blockchain-based infrastructure }\end{array}$ & $\begin{array}{l}\text { Custody and } \\
\text { settlement } \\
\text { Private } \\
\text { markets }\end{array}$ \\
\hline $\begin{array}{l}4 \\
\text { Fully decentralized } \\
\text { Uncertain }\end{array}$ & $\begin{array}{l}\text { - Blockchain replaces centrally controlled infrastructure with fully decentralized } \\
\text { solutions } \\
\text { - Direct engagement in digital asset transactions for organizations and individuals } \\
\text { - Legal and regulatory frameworks support asset ownership and transfers via } \\
\text { distributed ledgers } \\
\text { - Disintermediation of legacy infrastructure owners }\end{array}$ & $\begin{array}{c}\text { Open, P2P } \\
\text { blockchain-powered } \\
\text { economy } \\
\text { Digitally issued } \\
\text { fiat currency }\end{array}$ \\
\hline
\end{tabular}

Table 3. Blockchain Waves Source: Url-10, 2019 


\section{Conclusion}

Distributed ledgers named blockchain have the ability to securely digitize many current operations in economics and finance, and legal and government services. Blockchain can be defined as a decentralized public ledger, which records transactions between users in a permanent, secure and verifiable way. The important thing is that blockchain can be programmed to record not only financial transactions, but anything of value. There is a growing realization that blockchain technology will bring a radical shift of especially on financial assets. It is no doubt that the financial sector is at the forefront in adoption of blockchain technology. Blockchain is rapidly revolutionizing the global economy. The potential effect of blockchain technology - distributed ledgers - on the society and the global economy are hugely important, as they promise to always have an optimistic impact. Actually the potential benefits of the blockchain are more than just economic and the technological capacity of the blockchain is already being harnessed to address real world problems by specific groups.

Only nine years after the first Bitcoin white paper, blockchain technology is now studied by companies and governments to find possible use cases for efficiency and can possibly trigger the third industrial revolution. On the other hand, debate over blockchain's promise, as well as its limitations, is ongoing. Blockchain technology, in all its forms, continues to evolve rapidly. It is widely accepted that Blockchain technology future looks bright and attractive in part because of the way governments, developers, firms and investors are investing big as they seek to spur innovations and applications. The opportunities that blockchain offers need to be developed and governed wisely, with upfront and continual management of unintended consequences and downside risks. Blockchain is the kind of technological breakthrough that has the potential to make global changes. Its predicted impact on the world economy is big enough that some of the countries and biggest companies are already preparing for it.

\section{References}

- Ateniese G. Magri B., Venturi D., Andrade E., 2017. "Redactable Blockchain - or - Rewriting History in Bitcoin and Friends", 2nd IEEE European Symposium on Security and Privacy-EuroS\&P.

- Collomb A., Sok K., 2016. "Blockchain / Distributed Ledger Technology (DLT) What Impact On Financial Sector?", Digiworld Economic Journal no. 103, third quarter, p.93.

- Glaser F., 2017. "Pervasive Decentralisation of Digital Infrastructures: A Framework for Blockchain Enabled System and Use Case Analysis, Proceedings of the 50th Hawaii International Conference on System Sciences, Frankfurt.

- $\quad$ Liebenau, J., Elaluf-Calderwood, S.M., 2016. "Blockchain Innovation Beyond Bitcoin and Banking", Available at SSRN 2749890.

- $\quad$ Lindman J.,Rossi M.,Tuunainen V.K., 2017. "Opportunities and risks of Blockchain Technologies in Payments- a Research Agenda", Proceedings of the 50th Hawaii International Conference on System Sciences, pp 1533-1542.

- $\quad$ Ljutic A., McPhee C., 2017. "Editorial: Blockchain", Technology Innovation Management Review vol. 7 issue 10.

- Mainelli, M., Smith, M., 2015. "Sharing ledgers for sharing economies: an exploration of mutual distributed ledgers (aka blockchain technology) ", The Journal of Financial Perspectives 3, (3), pp. 38-69.

- Nakamoto, S., 2008. "Bitcoin: A Peer-to-Peer Electronic Cash System"

- $\quad$ Risius M., Shoprere K., 2017. "A Blockchain Research Framework: What We (don't) Know, Where We Go from Here, and How We Will Get There", Business \& Information Systems Engineering.

- Sun J. Yan J., Zhang K., 2016. "Blockchain-based sharing services: What blockchain technology can contribute to smart cities", Financial Innovation 2:26.

- Swan M., 2015. Blockchain: Blueprint For New Economy, O’reilly Media Inc.

- Swan M., 2017. "Anticipating the Economic Benefits of Blockchain", Technology Innovation Management Review vol. 7 issue 10.

- Tschorsch F., Scheuermann B., 2016. "Bitcoin and beyond: A technical survey on decentralized digital currencies", IEEE Communications Surveys \& Tutorials 18 (3):2084-2123.

- World Economic Forum (Wef), 2018. "Building Block (chains) For A Better Planet".

- Zimmermani 2016. The Evolution of Fintech, The New York Times, https://www.nytimes.com/2016/04/07/business/dealbook/the-evolution-of-fintech.html.

- Url-1, https://whatis.techtarget.com/definition/open-source, Alıntı Tarihi: 05.04.2019

- Url-2, https://www.weforum.org/reports/the-future-of-financial-infrastructure-an-ambitious-look-at-howblockchain-can-reshape-financial-services, 2016 
- Url-3, http://www.economist.com/news/leaders/21677198-technology-behind-bitcoin-could-transform-howeconomy-works-trust-machine, 2015

- Url-4, https://101blockchains.com/history-of-blockchain-timeline/\#prettyPhoto, Alıntı Tarihi: 03.04.2019

- Url-5, https://www.worldcryptoindex.com/creators/w-scott-stornetta/, Alıntı Tarihi: 04.04.2019

- Url-6, https://www.worldcryptoindex.com/blockchain/, Alıntı Tarihi: 05.04.2019

- Url-7, https://blockgeeks.com/guides/hyperledger/, Alıntı Tarihi: 06.04.2019

- Url-8, http://www.lteconomy.it/blog/2018/12/09/blockchain-economy-impact-and-future/, 2018

- Url-9, https://cryptobriefing.com/blockchain-adoption-study/, 2018

- Url-10, https://www.oliverwyman.com/content/dam/oliver-wyman/v2/publications/2016/jul/joint-report-byjp-morgan-and-oliver-wyman-unlocking-economic-advantage-with-blockchain-A-Guide-for-AssetManagers.pdf, Alıntı Tarihi: 06.04.2019 